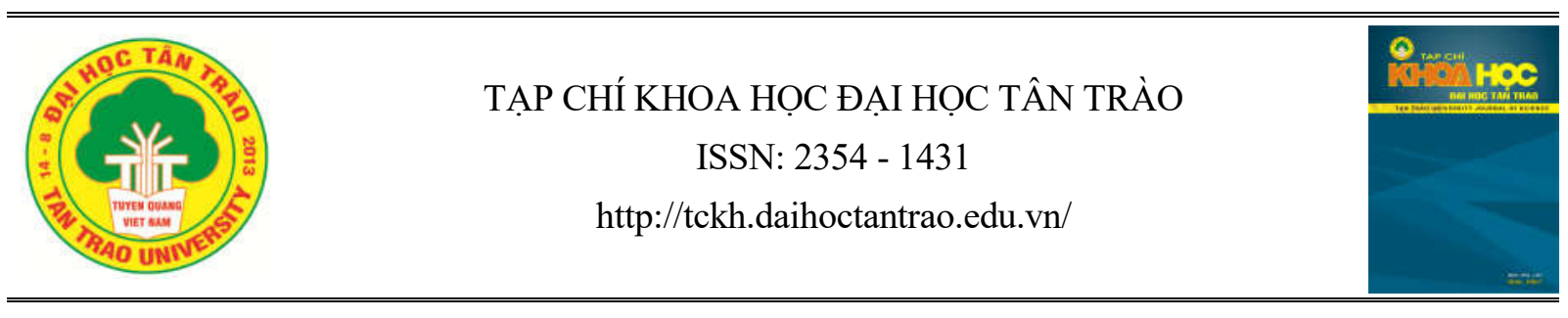

\title{
Sử dụng một số nguyên lí của toán rời rạc vào bài toán đếm bồi dưỡng sinh viên Olympic
}

\author{
Lê Thiếu Tráng ${ }^{a} *$ \\ ${ }^{a}$ Truò̀ng Đại hoc Tân Trào \\ *Email: lttrang0466@tuyenquang.edu.vn
}

\section{Thông tin bài viết}

Ngày nhận bài:

07/11/2018

Ngày duyệt đăng:

$10 / 12 / 2018$

\section{Tù khoá:}

Sinh viên; tổ hơp; bài toán đếm; nguyên lí; qui tắc;

rò̀i rạc

\section{Tóm tắt}

Toán rời rạc là một dạng toán khó và có vai trò quan trọng trong việc rèn luyện kĩ năng giải toán và giải quyết các vấn đề trong thực tiễn cho sinh viên. Các bài toán rời rạc được coi trọng trong chương trình môn toán phổ thông và đại học, cao đẳng của nhiều nước trên thế giới. Ở nước ta, do nhiều nguyên nhân khác nhau, dạng toán này còn chưa đề cập nhiều trong chương trình, chủ yếu được bổ sung cho học sinh giỏi thi các đội tuyển toán. Tuy nhiên, nếu không nắm được mạch kiến thức và phân loại đầy đủ, sinh viên trong đội tuyển Olympic toán cũng làm chưa tốt dạng toán này. Do vậy, việc trang bị kiến thức từ cơ bản đến nâng cao giúp cho sinh viên có thể giải quyết tốt dạng toán này.

\section{Một số kiến thức cơ bản về tổ hợp}

\section{Qui tắc đếm}

a. Qui tắc cộng: Một công việc được thực hiện theo một trong $k$ phương án $A_{1}, A_{2}, \ldots, A_{k}$.

Có $n_{1}$ cách thực hiện phương án $A_{1}, n_{2}$ cách thực hiện phương án $A_{2}, \ldots, n_{k}$ cách thực hiện phương án $A_{k}$. Khi đó có $n_{1}+n_{2}+\ldots+n_{k}$ cách thực hiện một trong các phương án trên.

Quan điểm tập hợp: Nếu tập hợp hữu hạn $X$ là hợp của $n$ tập hợp đôi một rời nhau $X_{1}, X_{2}, \ldots, X_{n}$ thì $|X|=\left|X_{1}\right|+\left|X_{2}\right|+\ldots+\left|X_{n}\right|$,

( $|X|$ là số phần tử của tập hợp $X$ ).

Ví dụ 1: Từ tập $X=\{a, b, c\}$, lập được bao nhiêu số tự nhiên, mỗi số có các chữ số khác nhau?

Giải: Lập phương án đếm: Đếm loại có 1 chữ số, loại có 2 chữ số và loại có 3 chữ số.

$X_{1}$ là tập hợp loại có 1 chữ số

$$
\Rightarrow X_{1}=\{a, b, c\} \Rightarrow\left|X_{1}\right|=3 \text {. }
$$

$X_{2}$ là tập hợp loại có 2 chũ số

$$
\Rightarrow X_{2}=\{\overline{a b}, \overline{a c}, \overline{b a}, \overline{b c}, \overline{c a}, \overline{c b}\} \Rightarrow\left|X_{2}\right|=6 \text {. }
$$

$X_{3}$ là tập hợp loại có 3 chữ số

$\Rightarrow X_{3}=\{\overline{a b c}, \overline{a c b}, \overline{b c a}, \overline{b a c}, \overline{c a b}, \overline{c b a}\} \Rightarrow\left|X_{3}\right|=6$.

Vậy có tất cả: $\left|X_{1}\right|+\left|X_{2}\right|+\left|X_{3}\right|=3+6+6=15$ số thỏa mãn bài toán.

b. Qui tắc nhân: Một công việc được thực hiện bao gồm $k$ công đoạn $A_{1}, A_{2}, \ldots, A_{k}$.

Công đoạn $A_{1}$ có $n_{1}$ cách thực hiện; Mỗi cách thực hiện công đoạn $A_{1}$ có có $n_{2}$ cách thực hiện công đoạn $A_{2} ; \ldots$... Mỗi cách thực hiện công đoạn $A_{1}$, $A_{2}, \ldots, A_{k-1}$ có $n_{k}$ cách thực hiện công đoạn $A_{k}$. Khi đó công việc được thực hiện bởi $n_{1} \cdot n_{2} \ldots n_{k}$ cách.

Quan điểm tập hợp: Nếu $X_{1}, X_{2}, \ldots, X_{n}$ là các tập hợp hữu hạn thì: 


$$
\left|X_{1} \times X_{2} \times \ldots \times X_{n}\right|=\left|X_{1}\right| \cdot\left|X_{2}\right| \ldots\left|X_{n}\right|,
$$

$\left(X_{1} \times X_{2} \times \ldots \times X_{n}\right.$ là tích Đề các của $n$ tập hợp $)$.

Ví dụ 2: Một chiếc cặp số, mã khóa là một bộ 3 số kết hợp của 3 vòng số, mỗi vòng gồm 10 số: $0,1, \ldots, 9$. Một người quên mã khóa, hỏi người đó phải thử nhiều nhất bao nhiêu mã khóa thì mới có thể mở được cặp số đó?

Giải: $\quad$ Gọi $\quad$ tập $\quad X=\{0,1,2,3,4,5,6,7,8,9\} \Rightarrow$ $|X|=10$. Một mã khóa có dạng $\overline{a b c}$, vì a, b, c đều có 10 lựa chọn từ $X$, nên số mã khóa là: $|X \times X \times X|=|X| .|X| \cdot|X|=10^{3}=1000$.

Vậy người đó phải thử tối đa 1000 mã khóa mới có thể mở chiếc cặp số đó.

\section{Một số khái niệm và công thức tổ hợp thường dùng}

a. Hoán vị không lặp: Cho tập hợp $X$ có $n$ phần tử $(n \geq 1)$, mỗi cách xếp thứ tự $n$ phần tử đó ta được một hoán vị (không lặp) các phần tử của tập $X$.

Số các hoán vị không lặp của tập hợp $X$ là: $P_{n}=n$ !

Ví dụ 3: Có 8 học sinh, trong đó gồm 4 nam và 4 nữ. Có bao nhiêu cách xếp 8 học sinh đó vào hai chiếc bàn, mỗi bàn có 4 chỗ ngồi trong hai trường hợp:

a) Các học sinh được xếp tùy ý; b) Học sinh nam một bàn và học sinh nữ ngồi một bàn.

Giải: a) Nếu các học sinh được xếp tùy ý: Đó chính là số các hoán vị của 8 phần tử, nên có:

$P_{8}=8 !=40320$ cách xếp.

b) Xếp 4 học sinh nam vào một bàn, 4 học sinh nữ vào bàn còn lại. Mỗi cách xếp một bàn đều là hoán vị của 4 phần tử, sau đó đảo thứ tự 2 bàn, nên số cách xếp là: $2.4 ! .4 !=1152$.

b. Hoán vị lặp: Cho tập hợp $X$ có $n$ phần tử $(n \geq 1)$, mỗi cách xếp thứ tự $n$ phần tử, trong đó phần tử $n_{1}$ lặp lại $k_{1}$ lần, phần tử $n_{2}$ lặp lại $k_{2}$ lần,..., phần tử $n_{p}$ lặp lại $k_{p}$ lần, với $k_{1}+k_{2}+\ldots+k_{p}=n$, gọi là một hoán vị lặp của $n$ phần tử của $X$.
Số các hoán vị lặp của tập hợp $X$ là: $P_{n}=\frac{n !}{k_{1} ! k_{2} ! \ldots k_{p} !}$

Ví dụ 4: Từ chữ $B E N Z E N$, có thể lập được bao nhiêu từ khác nhau?

Giải: Có 6 mẫu tự, được xếp vào 6 vị trí, trong đó chữ $\mathrm{E}$ và $\mathrm{N}$ đều được lặp lại 2 lần, nên số các từ khác nhau là: $\frac{6 !}{2 ! .2 !}=180$.

\section{c. Hoán vị vòng tròn của các phần tử phân biệt}

Cho tập hợp $X$ có $n$ phần tử $(n \geq 1)$, mỗi cách xếp $n$ phần tử đó trên một đường tròn ta được một hoán vị tròn của $n$ phần tử của $X$.

Số hoán vị tròn của n phần tử là : $\frac{P_{n}}{n}=(n-1)$ !

Ví dụ 5: Có 6 người ngồi họp trên một bàn tròn, trong đó có cử một người điều hành. Hỏi có bao nhiêu cách xếp chỗ ngồi khác nhau?

Giải: Trừ vị trí của người điều hành, còn 5 người được xếp vào 5 vị trí còn lại. Vì có thể cử bất kì ai làm người điều hành, nên số cách xếp là: $\frac{P_{6}}{6}=5 !=120$.

d. Chinh hợp không lặp: Cho tập hợp $X$ có $n$ $(n \geq 1)$ phần tử, mỗi cách xếp thứ tự $k$ phần tử $(1 \leq k \leq n)$, gọi là một chỉnh hợp (không lặp) chập $k$ của $n$ phần tử của $X$.

Số các chỉnh hợp không lặp chập $k$ của $n$ phần tử của $X$ là: $A_{n}^{k}=\frac{n !}{(n-k) !}$.

Ví dụ 6: Từ 10 điểm phân biệt, có thể lập được bao nhiêu vectơ khác vectơ-không?

Giải: Cứ hai điểm phân biệt, chẳng hạn $A, B$ ta lập được hai vectơ khác vectơ-không là $\overrightarrow{A B}$ và $\overrightarrow{B A}$, do đó có tất cả: $A_{10}^{2}=\frac{10 !}{(10-2) !}=90$ vectơ khác vectơ-không.

e. Chinh hợp lặ: Cho tập hợp $X$ có $n$ phần tử $(n \geq 1)$, mỗi cách xếp thứ tự $k$ phần tử $(1 \leq k \leq n)$, trong đó mỗi phần tử có thể lặp lại hữu hạn lần, gọi là 
một chỉnh hợp lặp chập $k$ của $n$ phần tử của $X$. Số các chỉnh hợp lặp chập $k$ của $n$ phần tử của $X$ là: $A_{n}^{k}=n^{k}$.

Ví dụ 7: Từ tập $X=\{0,1,2,3,4,5,6\}$. Lập được bao nhiêu số tự nhiên, mỗi số gồm 3 chữ số?

Giải: Gọi số cần tìm là $\overline{a b c}$. Vì $a \neq 0$, nên a có 6 cách chọn, còn $\mathrm{b}$ và $\mathrm{c}$ đều được chọn từ 7 số đã cho. Vậy số các số thỏa mãn là: 6.72=294.

e. Tổ hợp không lặp: Cho tập hợp $X$ có $n$ phần tử $(n \geq 1)$, mỗi tập con $k$ phần tử $(1 \leq k \leq n)$ gọi là một tổ hợp (không lặp) chập $k$ của $n$ phần tử của $X$.

Số các tổ hợp không lặp chập $k$ của $n$ phần tử của $X$ là: $C_{n}^{k}=\frac{n !}{(n-k) ! k !}$.

Ví dụ 8: Một đề thi tổ hợp 30 câu gồm: 10 câu Toán, 10 câu Lý và 10 câu Hóa. Trong đó 10 câu Toán lấy trong ngân hàng đề có 50 câu, 10 câu Lý lấy trong ngân hàng đề có 40 câu, 10 câu Hóa lấy trong ngân hàng đề có 30 câu. Hỏi có bao nhiêu cách lập một đề thi như thế?

Giải: Số các đề thi thành lập được là: $C_{50}^{10} \cdot C_{40}^{10} \cdot C_{30}^{10}$.

f. Tổ hợp lặp: Cho tập hợp $X$ có $n$ phần tử $(n \geq 1)$, mỗi tập con $k$ phần tử $(1 \leq k \leq n)$, mỗi phần tử có thể lặp lại hữu hạn lần, gọi là một tổ hợp lặp chập $k$ của $n$ phần tử của $X$.

Số tổ hợp lặp chập $k$ của tập hợp $X$ là: $C_{n+k-1}^{k}$.

\section{Nguyên lý bù trừ}

Kí hiệu $|X|$ là số phần tử (hay lực lượng) của tập hợp hữu hạn $X$, giả sử $X_{i}, 1 \leq i \leq n$ là các tập hữu hạn thì ta có:

$$
\begin{aligned}
& \text { a. Nếu } \bigcap_{i=1}^{n} X_{i}=\varnothing \text { thì: }\left|\bigcup_{i=1}^{n} X_{i}\right|=\sum_{i=1}^{n}\left|X_{i}\right| \\
& \text { b.Nếu } \bigcap_{i=1}^{n} X_{i} \neq \varnothing
\end{aligned}
$$

thì:

$$
\begin{aligned}
& \left|\bigcup_{i=1}^{n} X_{i}\right|=\sum_{i=1}^{n}\left|X_{i}\right|-\sum_{1 \leq i<j \leq n}\left|X_{i} \cap X_{j}\right| . \\
& +\sum_{1 \leq i<j<k \leq n}\left|X_{i} \cap X_{j} \cap X_{k}\right|-\ldots . .+(-1)^{n-1} \bigcap_{i=1}^{n} X_{i} \mid
\end{aligned}
$$

Ví dụ 10: Một lớp có 50 học sinh, trong đó có 15 học sinh có học lực giỏi, 20 học sinh có hạnh kiểm tốt và 12 học sinh có cả học lực giỏi và hạnh kiểm tốt. Hỏi có bao nhiêu học sinh của lớp vừa không đạt học lực giỏi vừa không đạt hạnh kiểm tốt?

Giải:

Gọi $A$ là tập hợp học sinh của lớp $\Rightarrow|A|=50$;

G là tập hợp học sinh đạt học lực giỏi $\Rightarrow|G|=15$;

T là tập hợp học sinh đạt hạnh kiểm tốt $\Rightarrow|T|=20$.

Gọi $B$ là tập hợp học sinh của lớp vừa không đạt học lực giỏi vừa không đạt hạnh kiểm tốt. Từ giả thiết $\Rightarrow|G \cap T|=12$. Nếu gọi B là tập hợp các học sinh vừa không đạt học lực giỏi vừa không đạt hạnh kiểm tốt, thì:

$$
\begin{aligned}
& |A|=|B|+|G \cup T| \Leftrightarrow 50=|B|+|G|+|T|-|G \cap T| \\
\Leftrightarrow & 50=|B|+15+20-12 \\
\Rightarrow & |B|=50-(15+20-12)=27 .
\end{aligned}
$$

Vậy số học sinh vừa không đạt học lực giỏi vừa không đạt hạnh kiểm tốt là 27 học sinh.

\section{Sử dụng phương pháp ánh xạ}

Cùng với tập hợp, ánh xạ là một khái niệm rất quan trọng và rất cơ bản của toán học. Nó có mặt trong tất cả các lĩnh vực toán học. Khái niệm ánh xạ chính là sự mở rộng tự nhiên của khái niệm số học. Ta nhắc lại một số khái niệm cơ bản:

a. Định nghĩa: Một ánh xạ $f$ từ tập $X$ đến tập $Y$ là một quy tắc đặt tương ứng mỗi phần tử $X$ của $X$ với một và chỉ một phần tử $y$ của $Y$.

Phần tử $y$ được gọi là ảnh của $x$ qua ánh xạ $f$ và được kí hiệu là $y=f(x)$. Tập $X$ được gọi là tập nguồn (hay tập xác định) của $f$. Tập $Y$ được gọi là tập đích (hay tập giá trị) của $f$. 
Ánh xạ $f$ từ $\mathrm{X}$ đến $\mathrm{Y}$ được kí hiệu là: $f: X \rightarrow Y$

$x \mapsto y=f(x)$.

- Khi $X$ và $Y$ là tập con của tập số thực, ánh xạ $f$ được gọi là một hàm số xác định trên $X$.

- Cho $a \in X ; y \in Y$. Nếu $f(a)=y$ thì ta nói $y$ là ảnh của a và a là tạo ảnh của $y$ qua ánh xạ $f$. Mỗi phần tử a của $X$ đều có một ảnh duy nhất (là phần tử $f(a)$ ). Mỗi phần tử $y$ của $Y$ có thể có một hoặc nhiều tạo ảnh và cũng có thể không có tạo ảnh nào.

Tập $f(X)=\{y \in Y \mid \exists x \in X, y=f(x)\}$ gọi là tập ảnh của $f$. Nói cách khác, tập ảnh $f(X)$ là tập tất cả các phần tử của $Y$ mà có tạo ảnh.

\section{b. Phân loại ánh xạ}

+ Đơn ánh: Ánh xạ $f: X \rightarrow Y$ được gọi là đơn ánh nếu với $a \in X, b \in Y$ mà $a \neq b$ thì $f(a) \neq f(b)$, tức là hai phần tử phân biệt sẽ có hai ảnh phân biệt.

$f$ là đơn ánh $\Leftrightarrow$ với $a \in X, b \in Y$ mà $f(a)=f(b) \Rightarrow a=b$.

+ Toàn ánh: Ánh xạ $f: X \rightarrow Y$ được gọi là toàn ánh nếu với mỗi phần tử $y \in Y$ đều tồn tại một phần tử $x \in X$ sao cho $f(x)=y$.

$f$ là toàn ánh $\Leftrightarrow Y=f(X)$.

+ Song ánh: Ánh xạ $f: X \rightarrow Y$ được gọi là song ánh nếu nó vừa là đơn ánh vừa là toàn ánh.

$f$ là song ánh $\Leftrightarrow$ Với mỗi $y \in Y$, tồn tại duy nhất một phần tử $x \in X$ để $f(x)=y$.

+ Ánh xạ ngược của một song ánh: Cho $f: X \rightarrow Y$ là một song ánh. Khi đó, với mỗi $y \in Y$, tồn tại duy nhất một phần tử $x \in X$ để $f(x)=y$. Phần tử duy nhất $x \in X$ này được gọi là ảnh của phần tử qua ánh xạ ngược của $f$. Như vậy ta có định nghĩa: Ánh xạ ngược của $f$, được kí hiệu bởi $f^{-1}$, là ánh xạ từ $\mathrm{Y}$ đến $\mathrm{X}$ gán cho mỗi phần tử $y \in Y$ phần tử duy nhất $x \in X$ để $f(x)=y$. Như vậy: $f^{-1}(y)=x \Leftrightarrow f(x)=y$.

+ Ánh xạ tích: Cho $g$ là ánh xạ từ tập $A$ đến tập $B$ và $f$ là ánh xạ từ tập $B$ đến tập $C$. Nếu $g(a) \in B$ với mỗi $a \in A$ (tức là $g(A) \subset B$ ) thì ta có thể xác định một ánh xạ từ $A$ đến $C$ theo quy tắc sau: Đặt tương ứng mỗi phần tử $a \in A$ với phần tử $f(g(a)) \in C$. Ánh xạ này được gọi là ánh xạ hợp của ánh xạ $f$ và ánh xạ $g$, kí hiệu $f \circ g$.

Như vậy ta có: Nếu $g: A \rightarrow B$ và $f: B \rightarrow C$ và $g(A) \subset B$ thì ánh xạ hợp $f \circ g: A \rightarrow C$ được xác định bởi: $(f \circ g)(a)=f(g(a))$.

+ Ánh xạ hằng: Cho ánh xạ $f: X \rightarrow Y$, nếu mỗi $x \in X: y=f(x)=c$ gọi là ánh xạ hằng.

+ Ánh xạ đồng nhất: Cho ánh xạ $f: X \rightarrow Y$, nếu mỗi $x \in X: y=f(x)=x$ gọi là ánh xạ đồng nhất.

* Sử dụng ánh xạ vào phép đếm: Qua phép song ánh, ta hoàn toàn có thể ước lượng số phần tử của một tập hợp $\mathrm{A}$ nào đó thông qua một tập hợp $\mathrm{B}$ mà ta biết số phần tử của nó nhờ một phép tương ứng giữa $\mathrm{A}$ và $\mathrm{B}$.

Định lí: Cho $\mathrm{A}$ và $\mathrm{B}$ là hai tập hợp hữu hạn: Nếu có một đơn ánh $f: A \rightarrow B$ thì $|A| \leq|B|$; Nếu có một toàn ánh $f: A \rightarrow B$ thì $|A| \geq|B|$; Nếu có một song ánh: $f: A \rightarrow B$ thì $|A|=|B|$

Ví dụ 11: Có một nhóm người mà trong đó mỗi cặp không quen nhau có đúng hai người quen chung, còn mỗi cặp quen nhau thì không có người quen chung. Chứng minh số người quen của mỗi người là như nhau.

Giải:

- Nếu $a$ quen $b$ và tập các người quen của $a$ và $b$ (không kể $a, b$ ) lần lượt là $A$ và $B$. Mỗi người $a^{\prime}$ thuộc $A$ sẽ quen với một người duy nhất thuộc $B$ (do $a^{\prime}$ và $b$ không quen nhau, hơn nữa họ đã có một người quen chung là $a$ ). Tương tự mỗi người thuộc $B$ cũng quen với duy nhất một người thuộc $A$. Vậy tồn 
tại một song ánh đi từ $A$ tới $B$, tức $a$ và $b$ có số người quen bằng nhau.

- Nếu $a$ không quen $b$ thì tồn tại $c$ quen cả $a$ và $b$. Do đó số người quen của $a$ và $b$ bằng nhau do cùng bằng số người quen của $c$ (suy ra từ trên).

\section{II . Bài tập ứng dụng}

\section{Dạng 1: các bài toán đếm cơ bản}

Bài toán 1: Từ tập con của tập $X=\{0 ; 1 ; 2 ; 3 ; 4 ; 5 ; 6 ; 7 ; 8 ; 9\}$, lập các số tự nhiên thỏa mãn những điều kiện đặt ra.

Bài mẫu 1: Cho tập $X=\{0 ; 1 ; 2 ; 3 ; 4 ; 5 ; 6 ; 7 ; 8 ; 9\}$.

1) Từ $X$ lập được bao nhiêu số tự nhiên, mỗi số có 4 chữ số?

2) Từ $X$ lập được bao nhiêu số tự nhiên chã̃n, mỗi số có 5 chữ số khác nhau?

3) Từ $X$ lập được bao nhiêu số tự nhiên không lớn hơn 5789, mỗi số có 4 chữ số khác nhau?

4) Từ $X$ lập được bao nhiêu số tự nhiên chia hết cho 3, mỗi số có 3 chữ số khác nhau?

5) Từ $X$ lập được bao nhiêu số tự nhiên mỗi số gồm 15 chữ số, trong đó: số 1 có mặt hai lần, số 2 có mặt 3 lần, số 9 có mặt 3 lần, mỗi số khác có mặt một lần?

6) Từ $X \backslash\{0 ; 9\}$ lập được bao nhiêu số tự nhiên, mỗi số có 8 chữ số khác nhau? Tính tổng các số tìm được?

7) Từ $X$ lập được bao nhiêu số tự nhiên, mỗi số có 6 chữ số khác nhau? Tính tổng các số tìm được?

8) Từ $X \backslash\{0\}$ lập được bao nhiêu số tự nhiên, mỗi số có 7 chữ số khác nhau, số 1 và 2 không đứng cạnh nhau?

\section{Giải:}

1) + Cách 1: Gọi $n(x)$ là số cách chọn $\mathrm{x}$. Giả sử số cần tìm là $\overline{a b c d}$, trong đó $a \neq 0, b, c, d$ lấy từ $X . \quad$ Vì $\quad a \in X \backslash\{0\} \quad$ nên $\quad n(a)=9$; $n(b)=n(c)=n(d)=10$.

Vậy có tất cả: $9 \cdot 10^{3}=9000$ số thỏa mãn bài toán.

+ Cách 2: Mỗi số $a, b, c, d$ đều có 10 cách chọn, kể cả $a=0$, nên có 104 số như thế
Xét trường hợp $a=0$, có một cách chọn $a$, mỗi số $b, c, d$ đều có 10 cách chọn, nên có 103 cách chọn $b, c, d$. Vậy có tất cả: 104-103=9000 số 4-

10410 số thỏa mãn bài toán.

2) Gọi số cần tìm là $\overline{a b c d e}$, trong đó $a \neq 0, b, c, d, e$ lấy từ $X$.

Nếu $e=0 \Rightarrow$

$n(e)=1 ; n(a)=9, n(b)=8, n(c)=7, n(d)=6$.

Vậy trường hợp này có: 1.9.8.7.6= 3024 số thỏa mãn.

- Nếu $e \in\{2 ; 4 ; 6 ; 8\} \Rightarrow n(e)=4$. Do $a \in X \backslash\{0\}$ nên $n(a)=8, n(b)=8, n(c)=7, n(d)=6$.

Vậy trường hợp này có: 4.8.8.7.6= 10752 số thỏa mãn.

Do đó có tất cả: $3024+10752=13776$ số thỏa mãn bài toán.

3) Cách 1: Đếm trực tiếp: Gọi số cần tìm là $\overline{a b c d}$, trong đó $a \neq 0, b, c, d$ lấy từ $X$.

- Nếu $a \in\{1 ; 2 ; 3 ; 4\} \Rightarrow n(a)=4$, thì $b, c, d$ tùy ý $\Rightarrow n(b)=9, n(c)=8, n(d)=7$.

Trường hợp này có: 4.9.8.7=2018 số thỏa mãn.

- Nếu a $=5 \Rightarrow n(a)=1$,

$b \in\{0 ; 1 ; 2 ; 3 ; 4 ; 6\} \Rightarrow n(b)=6$, thì $c, d$ tùy ý $\Rightarrow$ $n(c)=8, n(d)=7$.

Trường hợp này có: 1.6.8.7=336 số thỏa mãn.

- Nếu $\quad \mathrm{a}=5, \quad \mathrm{~b}=7 \quad \Rightarrow n(a)=n(b)=1$, $c \in\{0 ; 1 ; 2 ; 3 ; 4 ; 6\} \Rightarrow n(c)=6, n(d)=7$.

Trường hợp này có: 1.1.6.7=42 số thỏa mãn.

- Nếu $\quad \mathrm{a}=5, \quad \mathrm{~b}=7, \quad \mathrm{c}=8 \quad$ thì $\mathrm{d}=9 \Rightarrow n(a)=n(b)=n(c)=n(d)=1$. Trường hợp này có 1 số thỏa mãn.

Vậy có tất cả: $2018+336+42+1=2397$ số thỏa mãn bài toán.

Cách 2: Dùng nguyên lí loại trừ: Đếm số có bốn chữ số khác nhau lấy từ $X$, trừ các số không thỏa mãn.

4) $\quad \mathrm{Ta}$ có: $0 ; 3 ; 6 ; 9 \equiv 0(\bmod 3) \quad$ (I);

$$
1 ; 4 ; 7 \equiv 1(\bmod 3)
$$

$2 ; 5 ; 8 \equiv 2(\bmod 3)$ 
Các bộ ba số có tổng chia hết cho 3 được thành lập như sau: Trong nhóm (I) có $C_{4}^{3}=4$ bộ; Trong nhóm (II) có 1 bộ; Trong nhóm (III) có 1 bộ; Kết hợp ba nhóm có: 4.3.3=36 bộ

Vậy có tất cả 42 bộ ba số có tổng chia hết cho 3 . Trong đó:

Có 12 bộ chứa số $0 \Rightarrow$ Số các số lập được từ các bộ này là: $12\left(P_{3}-P_{2}\right)=48$ số.

Có 30 bộ không chứa số $0 \Rightarrow$ Số các số lập được từ các bộ này là: $30 \cdot P_{3}=180$ số.

Vậy có tất cả: $48+180=228$ số thỏa mãn bài toán.

Chú ý: Bài toán này đa số các sách hướng dẫn đều làm theo cách lập các bộ ba số có tổng chia hết cho 3 . Cách này rất dài và dễ sai sót. Nếu làm theo đồng dư như trên sẽ đơn giản và hiệu quả hơn. Có thể áp dụng cho các bài toán tương tự.

5) Xét một số thỏa mãn bài toán, chẳng hạn: 110222345678999.

- Nếu tính cả số 0 đứng đầu thì đây là hoán vị của 15 chữ số, trong đó số 1 lặp 2 lần, số 2 và số 9 lặp 3 lần $\Rightarrow$ số các số đó là: $\frac{P_{15}}{2 ! 3 ! 3 !}$

- Xét số 0 đứng đầu, thì đây là hoán vị của 14 chữ số, trong đó số 1 lặp 2 lần, số 2 và số 9 lặp 3 lần $\Rightarrow$ số các số đó là: $\frac{P_{14}}{2 ! 3 ! 3 !}$. Vậy số các số thỏa mãn bài toán là: $\frac{P_{15}}{2 ! 3 ! 3 !}-\frac{P_{14}}{2 ! 3 ! 3 !}$.

6) Số các số cần tìm là số hoán vị của 8 số của $X \backslash\{0 ; 9\}$ bằng $P_{8}=8$ !.

Để tính tổng $S$ các số tìm được, ta phân tích theo hai cách sau:

Cách 1: Dùng tính chất của hoán vị liên tục từ 1 đến 8 :

Mỗi số $\mathrm{x}$ trong các số tìm được, luôn tồn tại duy nhất số x’ trong các số đó mà:

$\mathrm{x}+\mathrm{x}^{\prime}=99999999$ (chẳng hạn $\mathrm{x}=12345678$ thì $\left.x^{\prime}=87654321\right)$.

Có tất cả $\frac{P_{8}}{2}$ cặp $\left(x ; x^{\prime}\right)$ như vậy. Do đó tổng cần tìm là: $S=99999999 \cdot \frac{P_{8}}{2}$.
Cách 2: Gọi số cần tìm là $\overline{a_{1} a_{2} a_{3} a_{4} a_{5} a_{6} a_{7} a_{8}}$, trong đó $a_{i} \in X \backslash\{0 ; 9\}, i=\overline{1,8}$.

Ta có:

$\overline{a_{1} a_{2} a_{3} a_{4} a_{5} a_{6} a_{7} a_{8}}=$ $a_{1} \cdot 10^{7}+a_{2} \cdot 10^{6}+a_{3} \cdot 10^{5}+a_{4} \cdot 10^{4}+a_{5} \cdot 10^{3}+a_{6} \cdot 10^{2}+a_{7} \cdot 10+a_{8}$

Mỗi số $a_{i}, i=\overline{1,8}$ đều xuất hiện ở mỗi hàng $P_{7}$ lần (bằng số hoán vị 7 phần tử còn lại).

Do đó $S=P_{7} \cdot(1+2+\ldots+8) \cdot\left(10^{7}+10^{6}+\ldots+10+1\right)$.

7) Áp dụng cách 2 ở ý 6 . Gọi số cần tìm là $\overline{a_{1} a_{2} a_{3} a_{4} a_{5} a_{6}}$, trong đó $a_{i} \in X \backslash\{0 ; 9\}, i=\overline{1,6}$.

Ta có:

$\overline{a_{1} a_{2} a_{3} a_{4} a_{5} a_{6}}=a_{1} \cdot 10^{5}+a_{2} \cdot 10^{4}+a_{3} \cdot 10^{3}+a_{4} \cdot 10^{2}+a_{5} \cdot 10+a_{6}$.

Mỗi số $a_{i}, i=\overline{1,6}$ đều xuất hiện ở mỗi hàng $A_{8}^{5}$ lần (bằng số sắp xếp 5 phần tử lấy từ 8 phẩn tử còn lại vào 5 vị trí). Do đó

$$
S=A_{8}^{5} \cdot(1+2+\ldots+8) \cdot\left(10^{5}+10^{4}+\ldots+10+1\right) .
$$

8) Ta dùng nguyên lí loại trừ: Số các số có 7 chữ số khác nhau lập từ $X \backslash\{0\}$ là $A_{9}^{7}$.

Xét các số không thỏa mãn bài toán, chẳng hạn: $\overline{12 a b c d e}$. Hai số 1, 2 hoặc 2, 1 đứng cạnh nhau, có 6 vị trí như vậy. Mỗi vị trí như thế, còn 5 vị trí được lấy từ 5 trong 7 số còn lại, số các số đó là $A_{7}^{5}$. Vậy có tất cả: $2 \cdot 5 \cdot A_{7}^{5}=25200$ số thỏa mãn.

Vậy số các số cần tìm là: $A_{9}^{7}-2 \cdot 5 \cdot A_{7}^{5}=156240$.

Bài mẫu 2: Cho tam giác $\mathrm{ABC}$. Xét 3 đường thẳng song song $\mathrm{AB}, 4$ đường thẳng song song $\mathrm{BC}, 5$ đường thẳng song song $\mathrm{CA}$. Hỏi các đường thẳng này tạo thành:

1) Bao nhiêu tam giác?

2) Bao nhiêu hình thang (không phải hình bình hành)?

3) Bao nhiêu hình bình hành? 


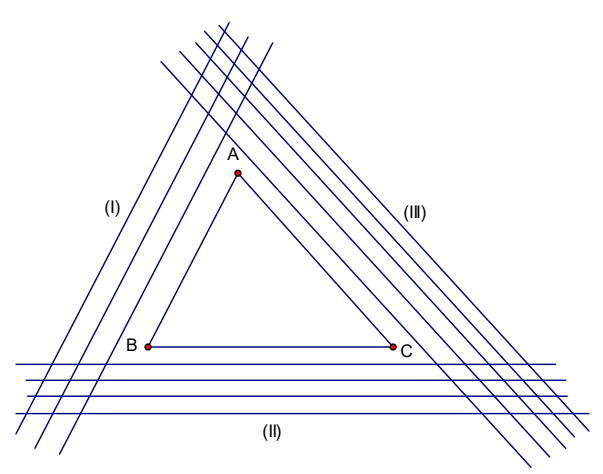

Giải: Gọi các đường thẳng song song $\mathrm{AB}$ là loại (I); Các đường thẳng song song $\mathrm{BC}$ là loại (II); Các đường thẳng song song $\mathrm{CA}$ là loại (III).

1) Mỗi đường thẳng loại $(\mathrm{I})$ cắt một đường thẳng loại (II), kết hợp với một đường thẳng loại (III) tạo nên một tam giác. Khi tráo đổi đỉnh và đáy thì các tam giác trùng nhau.

Vậy số tam giác tạo thành là: $C_{3}^{1} \cdot C_{4}^{1} \cdot C_{5}^{1}=60$.

2) Cứ hai đường thẳng của loại (I) kết hợp một đường loại (II) và một đường loại (III), tạo nên một hình thang (không phải hình bình hành). Có 3 trường hợp như vậy ở mỗi đỉnh tam giác. Do đó số hình thang tạo thành là:

$$
C_{3}^{2} \cdot C_{4}^{1} \cdot C_{5}^{1}+C_{4}^{2} \cdot C_{3}^{1} \cdot C_{5}^{1}+C_{5}^{2} \cdot C_{4}^{1} \cdot C_{3}^{1}=270
$$

3) Cứ hai đường thẳng loại (I) kết hợp hai đường thẳng loại (II) tạo nên một hình bình hành.

Có 3 trường hợp như vậy. Do đó số hình bình hành là: $C_{3}^{2} \cdot C_{4}^{2}+C_{4}^{2} \cdot C_{5}^{2}+C_{5}^{2} \cdot C_{3}^{2}=108$.

* Tổng quát bài toán khi có $\mathrm{m}, \mathrm{n}, \mathrm{p}$ đường thẳng lần lượt song song $A B, B C, C A$.

\section{Dạng 2: Các bài toán đếm nâng cao}

Bài toán 2: Một tập hợp có $\boldsymbol{n}$ phần tử, các phần tử của nó có những tính chất riêng biệt hoặc chung một số tính chất nào đó. Tìm số phần tử có chung những tính chất theo yêu cầu đặt ra.

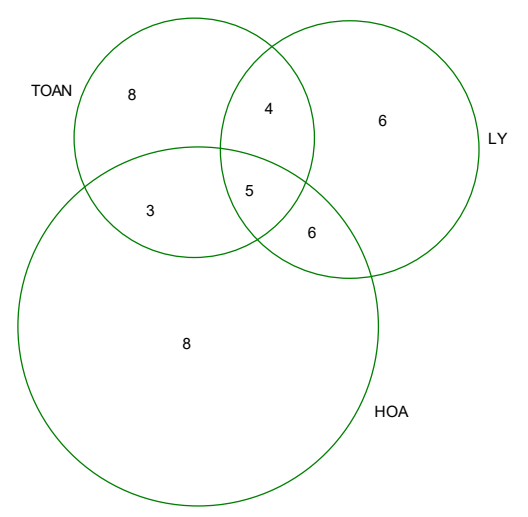

Bài mẫu 1: Một lớp có 40 học sinh, trong đó có 20 em giỏi Toán, 21 em giỏi Lí, 22 em giỏi Hoá; 8 em giỏi Toán và Hoá, 11 em giỏi Lí và Hoá, 5 em giỏi cả ba môn. Biết rằng mỗi học sinh trong lớp giỏi ít nhất một môn. Tìm số học sinh giỏi cả hai môn Toán và Lý.

Giải:

Cách 1: Biểu diễn sơ đồ Ven:

Dùng sơ đồ Ven, biểu diễn phần tử các tập hợp

theo giả thiết, ta thấy số học sinh vừ giỏi Toán, vừa giỏi Lí là 9 học sinh.

Cách 2: Dùng nguyên lí bù trừ: Gọi $L, H, T$ lần lượt tập hợp các học sinh giỏi Toán, Lý, Hoá.

Giả thiết cho: $|T|=20 ;|L|=21 ;|H|=22$;

$|T \cap H|=8 ;|L \cap H|=11 ;|T \cap L \cap H|=5 ;$

$|T \cup L \cup H|=40$. Ta phải tìm $|T \cap L|$.

Ta có:

$$
\begin{aligned}
& |T \cup L \cup H|=|T|+|L|+|H|-|T \cap L| \\
& -|T \cap H|-|L \cap H|+|T \cap L \cap H|
\end{aligned}
$$

Hay $40=20+21+22-|T \cap L|-8-11+5 \Rightarrow|T \cap L|$

$=20+21+22-8-11+5-40=9$

Vậy có 9 học sinh giỏi cả hai môn Toán và Lý.

Bài mẫu 2: Cho tập $A$ gồm 16 số nguyên dương đầu tiên. Hãy tìm số nguyên dương $k$ nhỏ nhất có tính chất: Trong mỗi tập con có $k$ phần tử của $A$ đều tồn tại hai số phân biệt $a, b$ sao cho $a^{2}+b^{2}$ là số nguyên tố.

Giải: Giả sử $k$ là số nguyên dương sao cho trong mỗi tập con có $k$ phần tử của tập $A$ đều tồn tại hai số phân biệt $a, b$ sao cho $a^{2}+b^{2}$ là số nguyên tố. Ta xét tập $T$ gồm các số chẵn thuộc tập $A$.

Khi đó $|T|=8$ và với mọi $a, b \in T$, ta có $a^{2}+b^{2}$ là hợp số $\Rightarrow k \geq 9$.

Xét các cặp số sau:

$A=(1 ; 2) \cup(3 ; 4) \cup(5 ; 16) \cup(6 ; 15) \cup(7 ; 12) \cup(8 ; 13) \cup(9 ; 10) \cup(11 ; 14)$

Xét $T$ là một tập con của $A$ và $|T|=9$, khi đó theo nguyên lí Dirichlet, $T$ sẽ chứa ít nhất một cặp nói trên, hay nói cách khác trong $T$ luôn tồn tại hai số phân biệt $a, b$ sao cho $a^{2}+b^{2}$ là số nguyên tố. Vậy số $k$ nhỏ nhất cần tìm là $k=9$. 
Chú ý: 1) Vì giả thiết $a^{2}+b^{2}$ là số nguyên tố nên $a^{2}+b^{2}$ không thể là số chẵn hay $a, b$ phải khác tính chẵn, lẻ. Dựa vào đó ta xây dựng được tập $T$.

2) Để tìm được sự phân hoạch tập $A$ thành hợp của 8 cặp rời nhau như trên ta làm như sau: Ta liệt kê tất cả các số $a_{i} \in A, i=\overline{1,16}$ sao cho $i^{2}+a_{i}^{2},(i=\overline{1,16})$ là số nguyên tố. Từ đó ta có được sự phân hoạch trên, sự phân hoạch trên không phải là duy nhất.

Bài mẫu 3: Có bao nhiêu số tự nhiên khác nhau không vượt quá 1000 là bội của 10, 15, 35, 55 .

Đặt:

$$
\begin{aligned}
& S_{1}=\{1 \leq n \leq 1000, n \vdots 10\} ; S_{2}=\{1 \leq n \leq 1000, n \vdots 15\} \\
& S_{3}=\{1 \leq n \leq 1000, n \vdots 35\} ; S_{4}=\{1 \leq n \leq 1000, n \vdots 55\}
\end{aligned}
$$

Khi đó:

$$
\begin{aligned}
& \left|S_{1}\right|=\left[\frac{1000}{10}\right]=100 ;\left|S_{2}\right|=\left[\frac{1000}{15}\right]=66 ; \\
& \left|S_{3}\right|=\left[\frac{1000}{35}\right]=28 ;\left|S_{4}\right|=\left[\frac{1000}{55}\right]=18
\end{aligned}
$$

Ta có:

$S_{1} \cap S_{2}=\{1 \leq n \leq 1000, n \vdots 10$ và $n: 15\}=\{1 \leq n \leq 1000, n \vdots 30\}$

$S_{1} \cap S_{3}=\{1 \leq n \leq 1000, n \vdots 10$ và $n \vdots 35\}=\{1 \leq n \leq 1000, n \vdots 70\}$

$S_{1} \cap S_{4}=\{1 \leq n \leq 1000, n: 10$ và $n: 55\}=\{1 \leq n \leq 1000, n: 110\}$

$S_{2} \cap S_{3}=\{1 \leq n \leq 1000, n \vdots 15$ và $n \vdots 35\}=\{1 \leq n \leq 1000, n \vdots 105\}$

$S_{2} \cap S_{4}=\{1 \leq n \leq 1000, n: 15$ và $n: 55\}=\{1 \leq n \leq 1000, n: 165\}$

$S_{3} \cap S_{4}=\{1 \leq n \leq 1000, n \vdots 35$ và $n: 55\}=\{1 \leq n \leq 1000, n \vdots 385\}$

Vì vậy:

$\left|S_{1} \cap S_{2}\right|=\left[\frac{1000}{30}\right]=33 ;\left|S_{1} \cap S_{3}\right|=\left[\frac{1000}{70}\right]=14 ;\left|S_{1} \cap S_{4}\right|=\left[\frac{1000}{110}\right]=9 ;$ $\left|S_{2} \cap S_{3}\right|=\left[\frac{1000}{105}\right]=9 ;\left|S_{2} \cap S_{4}\right|=\left[\frac{1000}{165}\right]=6 ;\left|S_{3} \cap S_{4}\right|=\left[\frac{1000}{385}\right]=2$

Mặt khác:

$S_{1} \cap S_{2} \cap S_{3}=\{1 \leq n \leq 1000, n: 210\} ; S_{1} \cap S_{2} \cap S_{4}=\{1 \leq n \leq 1000, n: 330\}$ $S_{1} \cap S_{3} \cap S_{4}=\{1 \leq n \leq 1000, n: 770\} ; S_{2} \cap S_{3} \cap S_{4}=\{1 \leq n \leq 1000, n: 1155\}$

Nên:

$$
\begin{aligned}
& \left|S_{1} \cap S_{2} \cap S_{3}\right|=\left[\frac{1000}{210}\right]=4 ; \quad\left|S_{1} \cap S_{2} \cap S_{4}\right|=\left[\frac{1000}{330}\right]=3 \\
& \left|S_{1} \cap S_{3} \cap S_{4}\right|=\left[\frac{1000}{770}\right]=1 ; \quad\left|S_{2} \cap S_{3} \cap S_{4}\right|=\left[\frac{1000}{1155}\right]=0
\end{aligned}
$$

Do:

$$
\begin{aligned}
& S_{1} \cap S_{2} \cap S_{3} \cap S_{4}=\{1 \leq n \leq 1000, n: 2310\} \Rightarrow \\
& \left|S_{1} \cap S_{2} \cap S_{3} \cap S_{4}\right|=\left[\frac{1000}{2310}\right]=0
\end{aligned}
$$

Vậy:

$:\left|S_{1} \cup S_{2} \cup S_{3} \cup S_{4}\right|=\sum_{i=1}^{4} S_{i}-\sum_{\mid \leq k<j 4}\left|S_{i} \cap S_{j}\right|+\sum_{\mid \leq k<k<4}\left|S_{i} \cap S_{j} \cap S_{k}\right|-S_{1} \cap S_{2} \cap S_{3} \cap S_{4} \mid$ $=(100+66+28+18)-(33+14+9+9+6+2)+(4+3+1+0)-0=147$.

\section{Dạng 3: Các bài toán đếm sử dụng phương pháp ánh xạ}

Bài toán 3: Xây dựng ánh xạ để đếm số phần tử một tập hợp hữu hạn.

Bài mẫu 1: Trong các xâu nhị phân có độ dài $n$, gọi $a_{n}$ là số các xâu không chứa 3 số liên tiếp $0,1,0$ và $b_{n}$ là số các xâu không chứa 4 số liên tiếp $0,0,1,1$ hoặc $1,1,0,0$. Chứng minh rằng $b_{n+1}=2 a_{n}$.

Giải:

Ta gọi một xâu thuộc loại $A$ nếu nó không chứa 3 số liên tiếp $0,1,0$.

Một xâu thuộc loại $B$ nếu nó không chứa 4 số hạng liên tiếp $0,0,1,1$ hoặc 1, 1, 0, 0 .

Với mỗi xâu $X=\left(x_{1}, x_{2}, \ldots, x_{n}\right)$, ta xây dựng $f(X)=\left(y_{1}, y_{2}, \ldots, y_{n}, y_{n+1}\right)$ như sau:

$$
y_{1}=0 ; y_{k} \equiv x_{1}+x_{2}+\ldots+x_{k-1}(\bmod 2), \forall k \in\{2,3, \ldots, n+1\}
$$

Khi đó $X$ chứa 3 số liên tiếp $0,1,0 \Rightarrow f(X)$ chứa 4 số hạng liên tiếp $0,0,1,1$ hoặc $1,1,0,0$. Hay $X$ thuộc loại $A \Rightarrow f(X)$ thuộc loại $B$.

Vậy $f$ là một song ánh đi từ tập các xâu loại $A$ độ dài $\mathrm{n}$ đến tập các xâu loại $B$ độ dài $n+1$ mà bắt đầu bằng 0 .

Nhưng từ mỗi xâu $X$ thuộc loại $B$ ta nhận được một xâu $X$ cũng thuộc loại $B$ bằng cách đổi các phần tử của $X$ theo quy tắc $1 \rightarrow 0,0 \rightarrow 1$ nên số các xâu loại $B$ độ dài $n+1$ gấp đôi số các xâu loại $B$ độ dài $n+1$ mà bắt đầu bằng số 0 . Từ đó ta có điều phải chứng minh.

Bài mẫu 2: Gọi $M$ là số các số nguyên dương viết trong hệ thập phân có $2 n$ chữ số, trong đó có $n$ chữ số 1 và $n$ chữ số 2 . Gọi $N$ là số tất cả các số viết trong hệ thập phân có $n$ chữ số, trong đó chỉ có các chữ số 1,2 , 3,4 và số chữ số 1 bằng số chữ số 2 .

Chứng minh rằng $M=N=C_{2 n}^{n}$.

Giải: Ta có $M=C_{2 n}^{n}$. Ta chứng minh $M=N$.

Với mỗi số có $n$ chữ số gồm các chữ số $1,2,3,4$ và số chữ số 1 bằng số chữ số 2 , ta "nhân đôi”" thành số có $2 n$ chữ số theo quy tắc sau: 
Đầu tiên, hai phiên bản của số này được viết kề nhau thành số có hai chữ số

Sau đó các chữ số 3 ở $n$ chữ số đầu và các chữ số 4 ở $n$ chữ số sau được đổi thành chữ số 1 , các chữ số 3 ở $n$ chữ số sau và các chữ số 4 ở $n$ chữ số đầu được đổi thành chữ số 2 .

Ví dụ:

$1234142 \rightarrow 12341421234142 \rightarrow 12121221221112$.

Như thế, ta thu được một số có đúng $n$ chữ số 1 và n chữ số 2 .

Rõ ràng đây là một đơn ánh từ tập các số $n$ chữ số sang tập các số $2 n$ chữ số.

Để chứng minh đây là một song ánh, ta xây dựng ánh xạ ngược như sau: với mỗi số có $n$ chữ số 1 và $n$ chữ số 2 , ta cắt $n$ chữ số đầu và $n$ chữ số cuối rồi cộng chúng theo cột với quy tắc: $1+1=1,2+2=2,1+2=3$, $2+1=4$, và ta thu được một số có $n$ chữ số gồm các chữ số $1,2,3,4$ với số chữ số 1 bằng số các số 2 . Chẳng hạn:

$$
12121221221112 \rightarrow \begin{aligned}
& 1212122 \\
& 1221112 \\
& 1234142
\end{aligned} \rightarrow 1234142 .
$$

Như thế song ánh giữa hai tập hợp đã được thiết lập và ta có $M=N=C_{2 n}^{n}$.

Bài mẫu 3: Có bao nhiêu cách chọn $k$ số từ $n$ số nguyên dương đầu tiên sao cho với hai số bất kì $a, b$ được chọn ta luôn có $|a-b|>m$ (ở đây không quan tâm thứ tự chọn các số này).

Giải: Ta cần tìm số phần tử của tập $A=\left\{\left(a_{1} ; a_{2} ; \ldots ; a_{k}\right) \mid a_{i}<a_{i+1}-m, 1 \leq a_{i} \leq n\right\}$

Xét tập

$B=\left\{\left(b_{1} ; b_{2} ; \ldots ; b_{k}\right) \mid b_{i} \leq b_{i+1} ; 1 \leq b_{i} \leq n-(k-1) m\right\}$

Thiết lập một ánh xạ $f: A \rightarrow B$ :

$\left(a_{1} ; a_{2} ; a_{3} ; \ldots ; a_{k}\right) \rightarrow\left(a_{1} ; a_{2}-m ; a_{3}-2 m ; \ldots ; a_{k}-(k-1) m\right)$

Ta dễ dàng chứng minh đây là một song ánh.

$\Rightarrow|A|=|B|$ bằng số cách chọn $\mathrm{k}$ số từ $n-(k-1) m$ số mà không quan tâm thứ tự.
Kết quả là: $C_{n-(k-1) m}^{k}$.

\section{Kết luận}

Qua theo dõi đề thi khu vực, quốc gia, quốc tế cho học sinh và sinh viên, tôi nhận thấy các dạng toán rời rạc được cho đôi khi cũng rất cơ bản, đôi khi được tích hợp từ các bài toán cơ bản. Do đó, để học sinh giải quyết tốt dạng toán này, các em cần được trang bị kiến thức thật cơ bản, phân loại các dạng toán thật tốt thì các em mới xử lí được những bài toán phức tạp. Trong quá trình nghiên cứu và tổng hợp tài liệu, do khả năng và thời gian có hạn nên một số kết quả của chuyên đề mới dừng lại ở những kết luận ban đầu, một số vấn đề của chuyên đề có thể chưa được phát triển sâu và cách làm có thể chưa tối ưu. Vì vậy rất mong được sự quan tâm đóng góp ý kiến của các thầy cô giáo, các bạn đồng nghiệp để bổ sung tốt hơn trong chuyên đề.

\section{TÀI LIỆU THAM KHẢO}

1. Bộ Giáo dục và Đào tạo - Hội Toán học Việt Nam, Tuyển tập Tạp chí Toán học và Tuổi trẻ, Nxb Giáo dục, 2003.

2. Chuyên đề Trại hè Hùng Vương các tỉnh miền núi phía Bắc 2015-2017.

3. Chuyên đề Trại hè các tỉnh duyên hải đồng bằng Bắc bộ 2016-2017.

4. Diễn đàn MATHCOPE.ORG. Tuyển tập các chuyên đề Tổ hợp,

5. Nguyễn Đễ - Nguyễn Khánh Nguyên (dịch), Một số đề thi vô địch Toán Olympic các nước, Nxb Giáo dục, 1996.

6. Nguyễn Văn Nho, OLYMPIC toán học Châu Á Thái bình duoong, Nxb Giáo dục 2003.

7. Nguyễn Đức Nghĩa, Nguyễn Tô Thanh, Toán rời rạc, Nxb Giáo dục 1999.

8. Đoàn Quỳnh, Tài liệu chuyên toán Đại số 10, 11, Nxb Giáo dục, 2006. 


\section{Using some principles of discrete mathematics in counting problems for Olympic students}

\section{Le Thieu Trang}

\section{Article info}

Recieved:

07/11/2018

Accepted:

$10 / 12 / 2018$

Keywords:

Student; combination; counting problem;

principle; rule; discrete.

\begin{abstract}
Discrete mathematics is a challenging form of math and plays an important role in training the skill of math solving and problem solving in reality for students. Discrete problems are highly focused in the math syllabus of high schools, universities and colleges of many countries in the world. In our country, this form of mathematics has been insignificantly mentioned in the syllabus, and mainly taught for good students in Mathematics teams due to different reasons. However, if knowledge flow and classification are not mastered completely, even students in Mathematical Olympiad teams will face challenges in solving this form of math. Therefore, equipping students with basic and advanced knowledge will help them to master this form of math well.
\end{abstract}

